\title{
Pro-active Life Cycle Engineering Support Tools
}

\author{
J. Duflou ${ }^{1}$, W. Dewulf ${ }^{1}$, P. Sas ${ }^{1}$ \\ ${ }^{1}$ Dept. of Mechanical Engineering, Katholieke Universiteit Leuven, Belgium \\ Submitted by P. Vanherck (1), Leuven, Belgium
}

\begin{abstract}
The extending responsibility of manufacturers regarding their products' environmental impact has led to the development of a wide range of Life Cycle Engineering tools and techniques. However, their efficiency has yet been limited by the need for detailed input data, typically unavailable in the early conceptual phases of design, when most far-reaching improvements can be achieved. This problem particularly holds true in a design environment of one-of-a-kind products, such as in many automation and machine design projects, where no previous generation products are available to provide the required analysis feedback. This paper presents a set of pro-active support tools that are specifically developed for the early design phases and cover the analytical as well as the creative steps of design. On the one hand, Eco-Cost Estimation Relationships form the core of the Eco-PaS system, which estimates the technical parameters, needed as input for most assessment tools, as a function of the functional parameters available in the concept development phase. On the other hand, the Ecodesign Knowledge System classifies both knowledge and user situations according to a common set of domain models in order to supply, at any time, adequate Life Cycle Engineering guidance, tailored to the specific user situation.
\end{abstract}

\section{Keywords:}

Environmental, Conceptual Design, Life Cycle

\section{INTRODUCTION}

As a result of the increased environmental awareness throughout all levels of present-day society, the environmental responsibility of manufacturers has increased to cover all phases of the product life cycle. In order to cope with these increasing demands, industry has, over the last decade, started to apply the principles of Life Cycle Engineering (LCE): the total environmental impact of a product can only be optimally controlled when considered already during product development.

A wide range of LCE support tools has been developed over the years (See e.g. [1], 2]). Figure 1 gives an overview of the major types of holistic LCE tools, which cover the whole life cycle of the product, categorised on the basis of two criteria: type of feedback versus time of application. The figure reveals one of the major limits of conventional LCE support tools: a clear void exists for tools making use of the functional parameters available in the early concept development phase of the design process.

Two strategies can be followed to cope with this problem, as visualised in Figure 1. One strategy is to rework generic guidelines in order to make them sensitive to the problem situation (1). This approach forms the basis of the Ecodesign Knowledge System (EKS) presented in Section 2. Another strategy consists of estimating the technical parameters, needed as input for most assessment tools, as a function of the functional parameters available in the concept development phase (2). This approach forms the basis of the Eco-PaS system presented in Section 3. Both approaches focus on a different aspect of designing: while the latter is mainly analysis-oriented, the former supports the creative, ideagenerating steps of design.

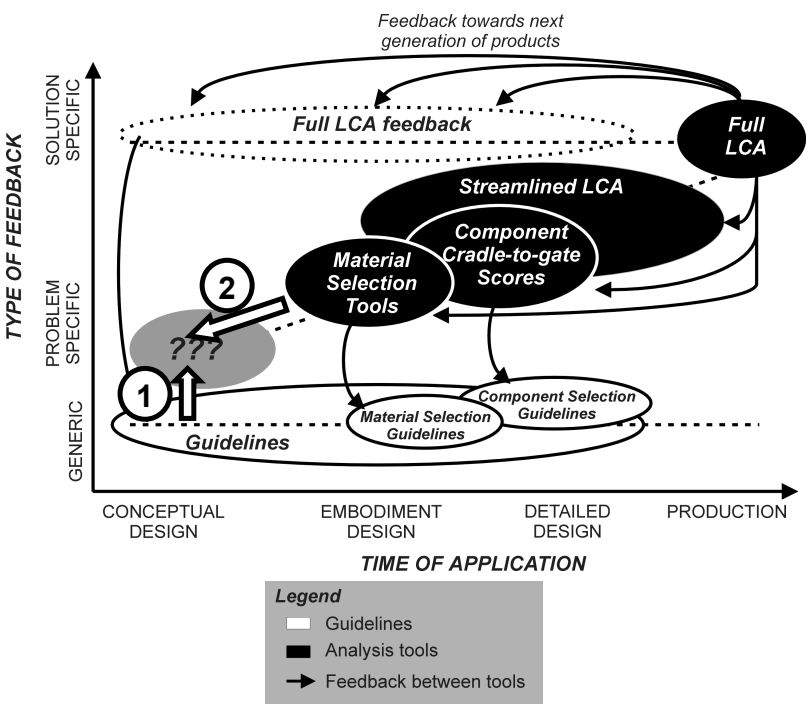

Figure 1 : Categorisation of holistic LCE Tools according to type of feedback and time of application

\section{THE ECODESIGN KNOWLEDGE SYSTEM (EKS)}

Design is an information intensive process. Ullman et al. [3] report a protocol study analysing the time use of five designers throughout a design process, and conclude that on average $19 \%$ of their time - with peaks to $36 \%$ in early conceptual design phases - is spent on information gathering activities. This order of magnitude is confirmed by other research teams and methods, such as by a 200 designers wide survey study by Court et al. [4]. 


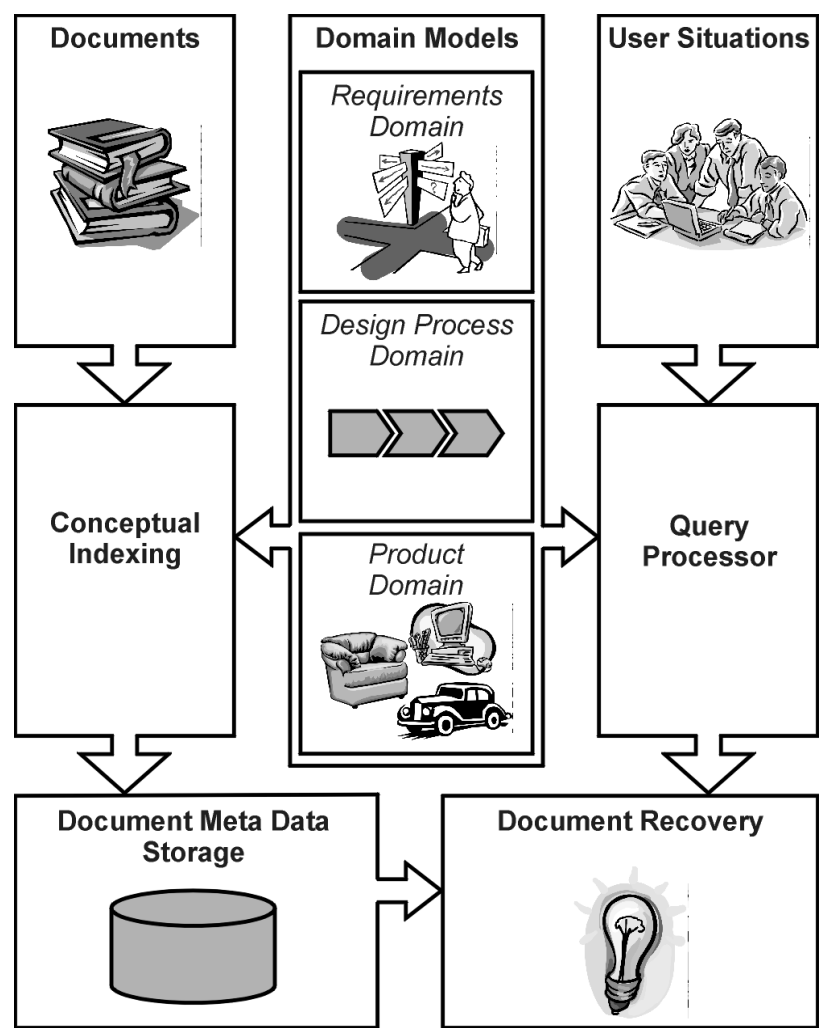

Figure 2: Concept of the Ecodesign Knowledge System
Mere availability of knowledge is, however, obviously insufficient. It is a well-known problem that many queries on knowledge repositories, such as the Internet, result in endless lists of potentially useful links, few of which prove to be relevant in the end. Moreover, the most relevant link is often not included. One important contributor to this problem is known to be the search actions being organised around (key)words contained in the target documents, rather than around concepts which these words denote. While synonymy - i.e. concepts being denoted by different words - causes queries to miss relevant documents, polysemy - i.e. words denoting different concepts - causes queries to hit irrelevant documents. As a solution to this problem, the Ecodesign Knowledge System makes use of predefined domain models for document as well as for query categorisation (see Figure 2). Domain models identify and describe concepts that exist in a domain as well as relations among these concepts. For the Ecodesign Knowledge System, models have been developed for three domains:

- the product domain, covering characterisations of the product to be designed. Concepts of the product domain model include both generic characterisations, such as 'plastic part' or 'electronics', as well as company or sector specific concepts, such as 'rail vehicle exterior door system' or 'bogie'. A partial product domain model tailored to the needs of a rail vehicle company is visualised in the top-left triangle of Figure 3 The relations between the concepts indicate what type of (more generic) components can be part of the more specific component.

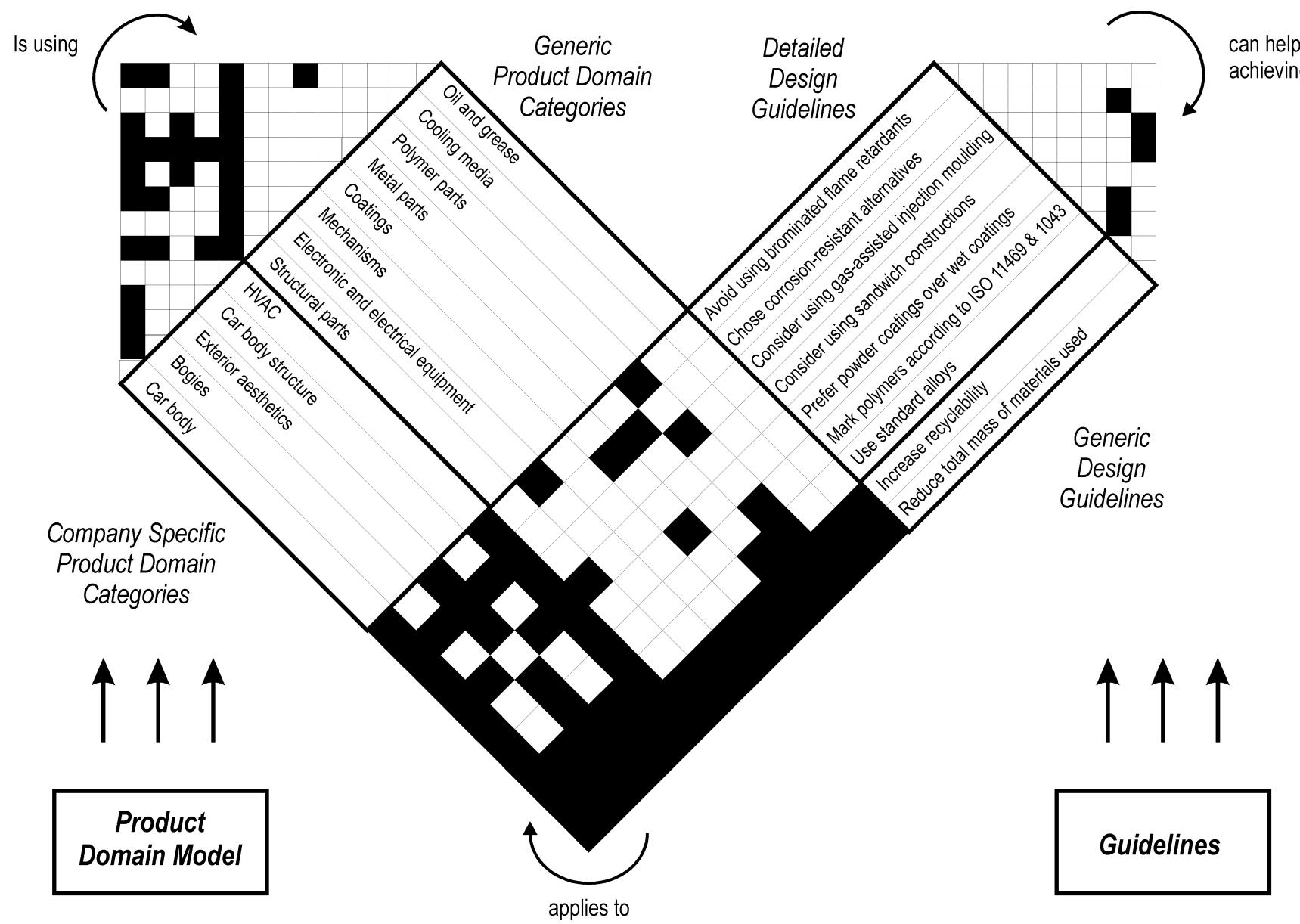

Figure 3: A hierarchy of guidelines mapped on a partial product domain model tailored to the needs of a rail vehicle manufacturer 
These relationships allow guidelines, linked to a generic concept, to be automatically linked to the more concrete, company-specific concepts. Similarly, links between product domain concepts and guidelines are inherited in the semantic guideline hierarchy represented in the right-hand side triangle;

- the requirements domain, covering the type of environmental requirements for the product life cycle. Concepts of this domain include e.g. Global Warming Potential or Recyclability;

- the design process domain, covering the design actions a user performs in converting requirements into an actual product. Concepts of this domain include the larger design phases as defined by Pahl and Beitz [5], such as conceptual design and embodiment design, as well as the elementary design activities as defined by Hubka [0], such as material selection, shape design, and tolerancing.

Together, they cover the basic elements from the ISO 9000 definition of design and development: set of processes that transforms requirements into specified characteristics and into the specification of a product, process or system, and are thus believed to sufficiently describe a user situation in order to retrieve the relevant pieces of information.

Within the framework of the current Ecodesign Knowledge System, manual classification has been presumed: when inserting a document into the system, a qualified user links the documents to the relevant concepts. This fits into the current organisation of ecodesign within large multi-national firms, where a dedicated ecodesign competence centre is gathering, structuring, and providing ecodesign knowledge for the whole company. A next stage of the research focuses, however, on the development of automatic classification of documents. The technique underlying this technology is based on semantic processing technology, recording the frequency of co-occurrence between words in a text, thus providing information about the meaning of the words used.

When retrieving documents from the repository, queries are formulated using concepts from the same domain models, which are then combined using various logical operators. In general, not only the concepts used in the query, but also those related to them through the domain model would be used to search conceptual descriptions of documents in the repository.

\section{PARAMETRIC ESTIMATION OF ENVIRONMENTAL IMPACT}

The second approach for pro-active LCE support builds on parametric cost estimating techniques, which are commonly used in e.g. aerospace industry to estimate the cost of projects and products early in the development process on the basis of Cost Estimating Relationships (CER's). CER's are mathematical expressions relating cost as the dependent variable to one or more independent cost driving variables [7], and are derived from historical, theoretical and literature data using e.g. regression analysis. In analogy to this definition, we propose to define Eco-Cost Estimating Relationships (E-CER's) as mathematical expressions relating an eco-cost as dependent variable to one or more independent eco-cost driving variables. In this framework, eco-costs need not necessarily be expressed in monetary units such as external costs [8] or willingness to pay [9], but can be expressed by any commonly used indicator such as Ecolndicator 99 [10, a Category Indicator Score [1T, or a product orented Environmental Performancefmdicator [12].

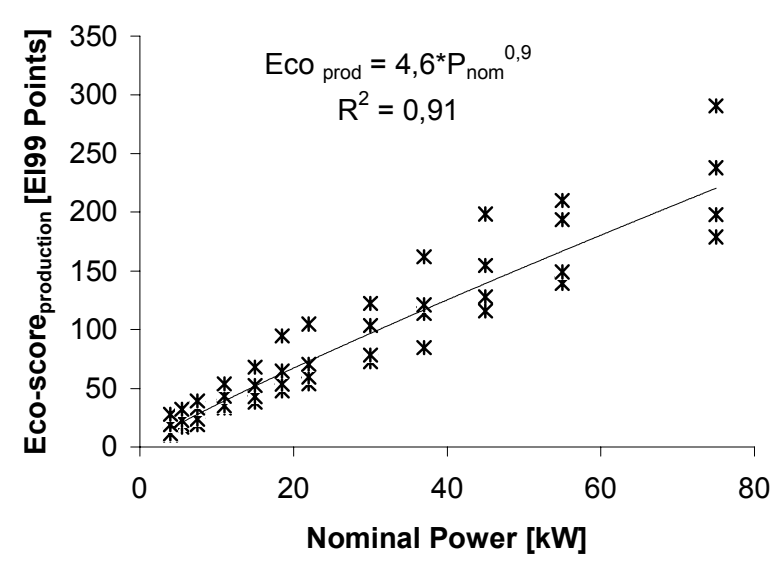

Figure 4: Eco-impact of the production of 3-phase AC motors of 5 to $75 \mathrm{~kW}$. The figure is based on a one supplier survey and only takes into account material production impacts

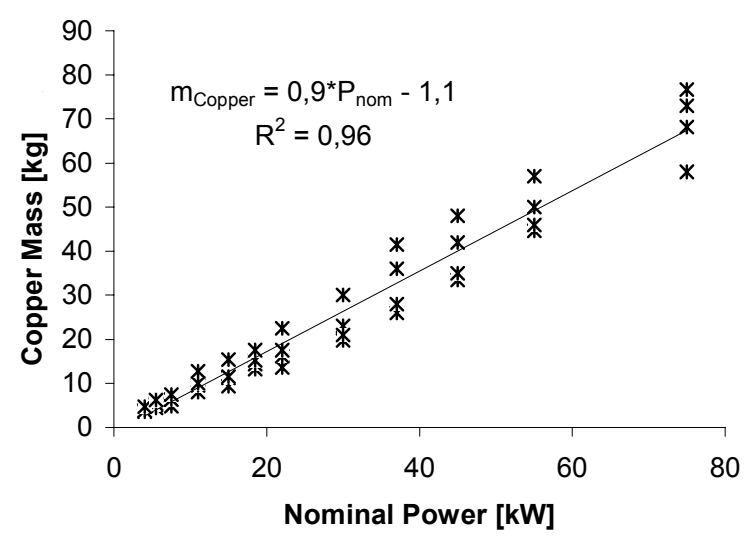

Figure 5: Mass of copper in 3-phase AC motors of 5 to $75 \mathrm{~kW}$.

Figure 4 exemplifies the results for an Eco-Indicator99 based E-CER for the production of 3-phase AC motors between 5 and $75 \mathrm{~kW}$, based on data from one producer [13. The $R^{2}$ value shown in the figure is already comfortably higher than the 0,8 lower limit used in e.g. NASA cost estimation procedures [7. Even better $R^{2}$ values can be obtained by splitting the total eco-cost according to eco-cost drivers such as the amount of individual materials, which do not necessarily follow similar types of regression curves.

For example, the linear regression curve of Figure 5 can be used to estimate the eco-impact of the motor's copper parts' production. The rule-of-thumb equation $\mathrm{m}_{\text {copper }}[\mathrm{kg}]$ $=P_{\text {nom }}[\mathrm{kW}]$ even provides a valuable approximation within the $5 \mathrm{~kW}-100 \mathrm{~kW}$ power range, still maintaining a coefficient of determination $R^{2}>0,9$.

E-CER's form the core of the Eco-PaS (Eco-efficiency Parametric Screening) tool, presented in Figure 6 EcoPaS supports the estimation of eco-impacts based on the functional parameters available in the concept development phase. It is based on the following principles:

- A design is usually conceived as an innovative combination of standard solutions to elementary functions. For example, the elementary function "illumination" is fulfilled by a selection out of the standard "lighting system" solutions.

- The system requires input in terms of functional descriptions and constraints (called Functional Parameters) instead of technical parameters. 


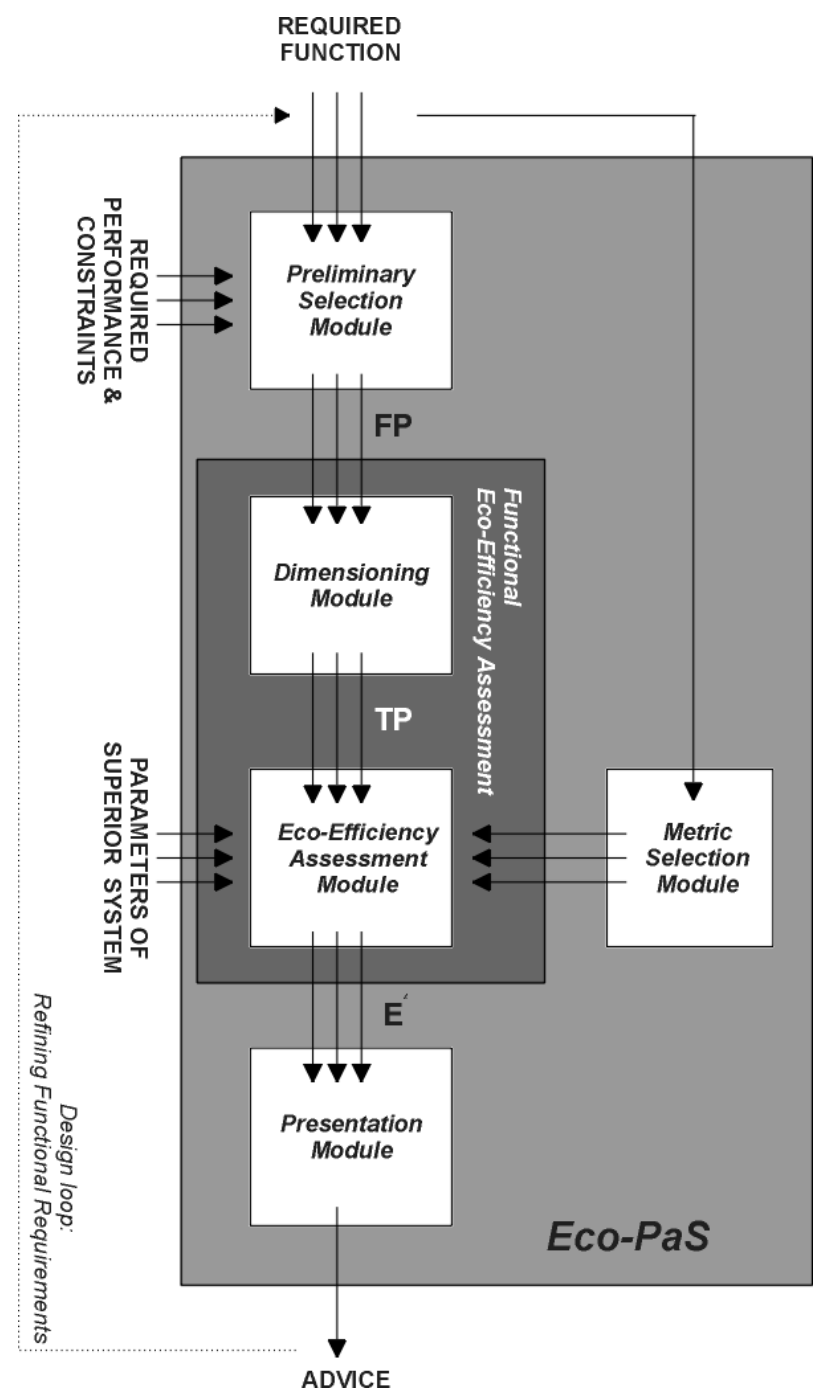

Figure 6: Principle of the Eco-PaS (Eco-efficiency Parametric Screening) system for Eco-Efficiency $\left(E^{2}\right)$ estimation based on functional parameters (FP) and intermediate technical parameters (TP)

- The system returns output in terms of quantified eco-efficiency performance indicators, even in the early conceptual phase.

- The system uses a convergent approach: all solutions are considered to be viable candidates unless proven inappropriate.

The core of the system is the Functional Eco-Efficiency Assessment making use of E-CER's. Both the direct variant (cf. Figure 4) resulting in eco-cost indicators as well as the indirect variant (cf. Figure 5) resulting in intermediate eco-cost drivers are possible. In the latter case, the intermediate eco-cost drivers are typically technical parameters such as the mass of individual material types, which are then easily converted into ecocost scores using the widely-used eco-cost material lists.

Auxiliary modules of Eco-PaS include:

- a preliminary selection module: the starting point of the method is the exact and abstract definition of the function required, e.g. illumination of a surface, as well as the definition of the required performance and the constraints, such as the required illumination [lux] and color. These constraints and performance requirements are referred to as functional parameters. The first input ("function required") serves as the basis for the Eco-PaS system to consult a database with known solution principles. A selection of technically feasible solution principles is then provided by an expert system, but can be modified through user intervention. In case of the illumination example, different types of lamps or lamp-luminaire combinations will be proposed;

- a metric selection module, allowing for selecting appropriate eco-efficiency performance metrics based on customer requirements, company policy, and legislation;

- a presentation module. The results of the Eco-PaS can be presented in a number of ways. For example, graphical representations allow showing for which combinations of functional parameters the the different solution principles are appropriate (i.e. for example more than $20 \%$ better than the other solutions).

\section{SUMMARY}

This paper presented two strategies to cope with the current lack of LCE support tools applicable in the conceptual design phase. On the one hand, an Ecodesign Knowledge System supplies problem-specific guidance based on mapping both available knowledge and user situations on three domain models. On the other hand, Eco-Cost Estimating Relationships are introduced as an extension to CER's. These E-CER's allow for estimating the environmental impact of design concepts based on a limited number of functional parameters.

\section{REFERENCES}

[1] Alting, L., Legarth, J., 1995, Life Cycle Engineering and Design, Annals of the CIRP, 44/2:569-580.

[2] Westkaemper, E., Alting, L., Arndt, G., 2000, Life Cycle Management and Assessment: Approaches and Visions Towards Sustainable Manufacturing, Annals of the CIRP, 49/2:501-522.

[3] Ullman, D., Dietterich, T., Stauffer, L., 1988, A Model of the Mechanical Design Process Based on Empirical Data, Artificial Intelligence in Engineering Design and Manufacturing, 2/1:33-52.

[4] Court, A., Culley, S., McMahon, C., 1993, The Information Requirements of Engineering Designers, Proceedings of ICED 1993, The Hague, August 17-19:1708-1716.

[5] Pahl, G., Beitz, W., 1996, Engineering Design - A Systematic Approach, Springer-Verlag, London.

[6] Hubka, V., 1988, Theory of Technical Systems, Springer-Verlag, Berlin.

[7] Brundick, B. (Ed.), 1996, Parametric Cost Estimation Handbook, US Navy, Arlington.

[8] European Commission DGXII, 1995, ExternE, Externalities of Energy, Vol. 1, EC, Brussels.

[9] Steen, B., 1999, A systematic approach to environmental priority strategies in product development (EPS). Version 2000 - General system characteristics, CPM, Gothenburg.

[10] Goedkoop, M., Effting, S., Collignon, M., 2000, The Eco-Indicator99 - Manual for Designers, Pré Consultants, Amersfoort.

[11] ISO 14042:2000, Environmental Management - Life Cycle Assessment - Life Cycle Impact Assessment, International Standard, ISO, Geneva.

[12] Dewulf, W., Duflou, J., Ander, A. (Eds.), 2001, Integrating Eco-Efficiency in Rail Vehicle Design, Leuven University Press, Leuven.

[13] ATB, 1995, Catalogue of A1 Standard three phase motors. 